Please quote as: Leimeister, J. M. \& Krcmar, H. (2005): Evaluation der Akzeptanz und des Nutzens einer systematisch entwickelten Virtuellen Community für Krebspatienten. In: Tagungsband der Konferenz Wirtschaftsinformatik, Bamberg. 


\title{
Evaluation der Akzeptanz und des Nutzens einer systematisch entwickelten Virtuellen Community für Krebspatienten
}

\author{
Jan Marco Leimeister, Helmut Krcmar \\ Technische Universität München
}

\begin{abstract}
Zusammenfassung: Virtuelle Communities (VC) bieten zeit- und ortsunabhängigen Zugang zu Informationen und Austausch mit Menschen in ähnlichen Lebenslagen. Besonders geeignet erscheinen sie daher für Patienten mit chronischen / lebensbedrohenden Krankheiten, da diese über besonders starke Informations- und Interaktionsbedarfe verfügen. Aufbauend auf Vorarbeiten zum bedarfs gerechten Aufbau der VC krebsgemeinschaft.de evaluiert dieser Beitrag die bei der systematischen Entwicklung zugrunde gelegten Designelemente und Erfolgsfaktoren anhand der Akzeptanz und des Nutzens des Angebots. Hieraus werden empirisch untermauerte Erkenntnisse zum systematischen Aufbau und Betrieb von VCs für Patienten im deutschen Gesundheitswesen gewonnen.
\end{abstract}

Schlüsselworte: Virtuelle Gemeinschaft, Virtuelle Community, Patienten, Community-Building, Evaluation.

\section{Rahmenbedingungen und Potentiale Virtueller Communities für Krebspatienten}

Krebserkrankungen sind mit ca. 338000 Neuerkrankungen jährlich die zweithäufigste Todesursache in Deutschland [Deut03]. Umso erstaunlicher ist, dass bis zum Jahr 2001 kaum Informations- und Interaktionsangebote für Patienten im deutschsprachigen Internet $\mathrm{zu}$ finden waren [Daum $\left.{ }^{+} 01\right]$. Ausgehend von dieser Erkenntnis wurde im Rahmen des $\operatorname{COSMOS}^{1}$-Forschungsprojektes eine VC für Krebspatienten entwickelt, eingeführt und betrieben. Dieser Beitrag behandelt aufbauend auf Vorarbeiten zum systematischen, bedarfsgerechten Aufbau der VC krebsgemeinschaft.de die Evaluation der Akzeptanz und des Nutzens des Angebots für Krebspatienten. Nach einer Darstellung der Ausgangssituation von Patienten wird auf die Potentiale von VCs für Patienten eingegangen. Daran schließt

\footnotetext{
Das Forschungsprojekt COSMOS (Community Online Services and Mobile Solutions) ist ein Verbundprojekt der Technischen Universität München und O2 (Germany) GmbH \& Co. OHG. Das Projekt wird vom Bundesministerium für Bildung und Forschung, FKZ 01 HW 0107 -0110, gefördert. Weitergehende Informationen sind zu finden unter http://www.cosmos-community.org.
} 
sich eine Zusammenfassung zentraler Design elemente eines bedarfsgerechten Angebots sowie deren Ausprägungen im Fall von krebsgemeinschaft.de an. Auf dieser Grundlage werden die Akzeptanz und der Nutzen des Angebots evaluiert und die Implikationen der Erkenntnisse dargestellt.

\subsection{Bedarfe von Krebspatienten}

Die Diagnose der Krebserkrankung trifft den Patienten meist völlig unerwartet. Die oft langwierigen Behandlungszyklen dominieren den Tagesablauf und die mittelfristige Lebensplanung. Arbeit und Privatleben bzw. Familie verlieren aufgrund dieser veränderten Lebenssituation an Priorität. Ausgelöst durch die lebensbedrohende Diagnose stürzt der Patient oftmals auch psychisch in eine Krise. Diese Krisen lösen einen starken Bedarf nach einem Sense-Making-Prozess der neuen Lebenssituation aus [Mada97]. Verstehen, was mit dem Betroffenen passiert, setzt Information über die Krankheit voraus. Doch der Wunsch nach harten Fakten ist nur ein Teil eines „Coping ““ -Prozesses (d. h. Verarbeiten und Bewältigen von Lebenskrisen). Neben dem Bedarf an Informationen kann es auch ein Verlangen nach Verständnis und emotionaler Unterstützung durch andere Betroffene sowie nach Interaktion mit diesen geben.

\subsubsection{Informationsbedarfe}

Krebspatienten entwickeln oft einen hohen Informationsbedarf, z. B. nach der Diagnose oder während einer Therapie [BiDe96; Hind ${ }^{+} 95$; McTh95; MiSu99]. Dieser Bedarf kann u. a. aus einer asymmetrischen Informationsverteilung zwischen Arzt und Patient resultieren.

Welche Art von Informationen von den Patienten im Detail nachgefragt wird, ze igen neuere Forschungsergebnisse über die Art der Bedürfnisse der Krebspatienten. Kaminski [Kami01] bspw. identifizierte unter anderem eine starkes Interesse an Informationen aus unterschiedlichen Bereichen, z. B. Auswirkungen der Erkrankung auf andere Lebensbereiche, wie Arbeit, Familie oder Sexualität. Mit diesen Fragen ist der behandelnde Arzt oftmals überfordert. Dabei geht es den Patienten nicht nur um me dizinische Kompetenz im engeren Sinne, sondern vor allem um Kommunikation und einen menschlichen Umgang mit dem individuellen Leiden der Einzelnen [Hill01]. Wechselseitige Akzeptanz, mehr Zuwendung, Empathie und Ganzheitlichkeit sowie eine höhere Qualität und bessere Zusammenarbeit zwischen den verschiedenen Akteuren im Behandlungsprozess werden als Problemfelder genannt.

2 Abgeleitet aus dem Englischen: „to cope“: mit einer Situation fertig werden, ihr gwachsen sein [SeUd93, S. 150f]. 


\subsubsection{Interaktionsbedarfe}

Oftmals ist auch im direkten Umgang mit betroffenen Menschen das Bedürfnis nach Empathie und zwischenmenschlicher Interaktion spürbar. Interaktion mit Gleichgesinnten/Menschen mit gleichem Schicksal kann eine große Rolle spielen [Forb01]. Die Bedarfe der Patienten beschränken sich nicht nur auf reine Fakten, vielmehr wollen sie, dass Informationen begründet und hinsichtlich ihres Krankheitsbildes ausgewertet und für ihre Lage interpretiert werden (vgl. hierzu auch [MiSu99; Moe196; SEKI00]. Um die neue Situation zu verarbeiten, das Wissen um Behandlungsmöglichkeiten oder Informationen über Heilungschancen zu diskutieren, braucht der Betroffene einen oder mehrere Gesprächspartner. Besonders interessiert ist er an Erfahrungen oder ähnlichen Fällen [BiDe96; Lieb ${ }^{+} 03$; Ma$\left.\mathrm{na}^{+} 02\right]$. Zwar ist im Falle einer Krebserkrankung nicht immer mit einem aktiven Umgang in Form von Informationssuche und Interaktion mit anderen Menschen zu rechnen. Dass aber Menschen, die aktive Krankheitsbewältigung mittels Informationssuche und Austausch betreiben, einen Vorteil hieraus ziehen können, ist in der Literatur mehrfach belegt (vgl. hierzu bspw. $\left.\left[\mathrm{McPh}^{+} 01\right]\right)$.

\subsection{Gesetzliche Rahmenbedingungen bei medizinischen Internetangeboten in Deutschland}

Das Gesundheitswesen ist in Deutschland ein hoch reglementierter Bereich. Beteiligte am Gesundheitswesen unterliegen qua ihrer Berufszugehörigkeit oder Anstellung bei einer bestimmten Einrichtung bestimmten Rechtsnormen. Darüber hinaus regeln weitere Gesetze den Gegenstandsbereich. Medizinische und PatientenInformationsangebote im Internet unterliegen den allgemeinen Anforderungen an Internetangebote. Es bestehen nur wenige Sonderregelungen, die beim Angebot medizinischer Inhalte zu beachten sind [Dier 03, S. 95]. Es sind insbesondere der Mediendienste-Staatsvertrag, das Teledienstegesetz. (TDG), sowie die Novellierungen im Rahmen des Gesetzes über die rechtlichen Rahmenbedingungen für den elektronischen Geschäftsverkehr (BGB1. I, S. 3721) zu berücksichtigen.

Besondere Beachtung verdienen insb. die folgenden Aspekte: Die Regelung der Haftung des Inhaltes fremder Websites (Verlinkung), die Berücksichtigung heilberufsrechtlicher Grenzen (in Form der Unterscheidung zwischen Information und Beratung), Aspekte des Datenschutzes in Zusammenhang mit Telediensten sowie Besonderheiten der Weitergabe von Fachinformationen an nicht autorisierte La ien. Diese rechtlichen Rahmenbedingungen haben dergestalt Einfluss auf Internetangebote in Deutschland, dass sie bspw. entsprechende Nutzer- und Nutzungsvereinbarungen sowie Disclaimer erfordern. Außerdem führen sie dazu, dass individuelle Beratungsangebote für Patienten über das Internet nicht zulässig sind und nur durch Informationsangebote annähernd ersetzt werden können. 


\subsection{Potentiale von Virtuellen Communities für Patienten}

Im Gesundheitswesen sind bisher bestehende Angebote für Patienten im allgemei nen und Krebspatienten im Besonderen meist an einen Ort oder an bestimmte (Öffnungs-) Zeiten gebunden. Eine Beratungsstelle ist zu festgesetzten Zeiten geöffnet - der Patient oder Angehörige muss sich physisch dorthin bewegen. Herkömmliche Selbsthilfegruppen treffen sich ebenfalls immer zu einer festgesetzten Zeit an einem physischen Ort, meist in einem Ballungsraum. Dementgegen sind Internetangebote und Virtuelle Communities hieran nicht gebunden und gerade für schwach besiedelte Gebiete interessant. Lange Anfahrtswege stellen nicht nur für Personen ohne eigenes Auto ein Problem dar, sondern auch für diejenigen, die aufgrund der Therapie oder durch Medikamente vorübergehend nicht am Straßenverkehr teilnehmen sollen.

\subsubsection{Aktualität, Anonymität und Bedarfsgerechtigkeit}

Die Aktualität von Informationen kann im Internet oftmals wesentlich höher sein als dies bei anderen Medien der Fall ist. Dies kann im Kontext gesundheitsbezogener Informationen (bspw. zu neuen Behandlungsansätzen, Versorgungsleistungen, etc.) bei entsprechend dringendem Interesse von Patienten ein großer Vorteil für das Medium sein. Neue Forschungsergebnisse oder aktuelle Entwicklungen sind über das Internet viel schneller verfügbar. Außerdem lässt sich oftmals im Internet eine größere Offenheit der Menschen feststellen, insbesondere wenn es bspw. um lebensbedrohende Krankheiten, schwie rige Situationen oder Tabu-Themen geht. Dies lässt sich unter anderem durch das Gefühl der Anonymität der Teilnehmer im Netz erklären. Die Bedarfsgerechtigkeit eines Internetangebots kann deutlich höher sein als bei Angeboten in anderen Medien, da der Nutzer die einzelnen Bestandteile nach seinen Wünschen einfach zusammenstellen kann, bspw. über welches Thema, wann und wie er sich informiert. Aus dem unterschiedlichen Nutzungsverhalten von Informationen in Abhängigkeit vom verwendeten Medium ergeben sich teilweise andere Anforderungen als an Texte für papierbasierte Medien.

\subsubsection{Interaktion, Empathie und Stärkung der Patienten}

Daneben bietet das Internet durch seine Dienste multilaterale Interaktionsmöglichkeiten. Ein Dienst sorgt dafür, dass Informationen verteilt und verwaltet werden und dass Kommunikation stattfinden kann. Er stellt eine Verbindung zwischen den Kommunikationspartnern her. Ein weiteres Merkmal der Interaktivität ist die Integration eines Feedback-Kanals sowie eine kollaborative Massenkommunikation, was bedeutet, dass alle Personen zugleich Sender- und Empfängerrollen übernehmen können [Döri03, S. 41f;RaLa93]. Die Interaktion in Virtuellen Gemeinschaften ermöglicht oftmals eine stark ausgeprägte Empathie unter den Mitgliedern sowie eine damit verbundene emotionale Integration in eine Gemein- 
schaft. Empathie lässt sich i. A. durch drei Eigenschaften charakterisieren [LeRu92, S. 234]: a) wissen, was eine andere Person fühlt, b) fühlen, was eine andere Person fühlt und c) mitfühlend antworten/agieren auf das Elend/Leid einer anderen Person. Beispiele hierfür lassen sich zahlreich in der Literatur finden [Pree99; Pree00; Pree01]. Die themenspezifische Interaktion der Teilnehmer generiert einen Informationspool hoher Objektivität und Glaubwürdigkeit [Schu99, S. 100; PeRo97, S. 244]. Die durch soziale Interaktion entstehende Erhöhung der Markttransparenz kann zu einer Informationsasymmetrie zugunsten der Mitglieder einer Gemeinschaft führen, da die Nachfrager nicht mehr primär auf die Informationen der Anbieter angewiesen sind [WiLi01, S. 288]. Zugleich können sich potentielle Kunden zu einer Gemeinschaft zusammenschließen, wodurch sie möglicherweise ein höheres Marktpotenzial und eine entsprechende Marktmacht erhalten könnten [LeSc01; Schu99, S. 99]. Angesichts des hoch reglementierten, nicht auf Wettbewerb ausgerichteten und sehr intransparenten deutschen Gesundheitssystems ist diese Folgerung jedoch leider als sehr visionär einzuschätzen.

\section{Zentrale Designelemente bedarfsgerechter Virtueller Communities für Patienten am Beispiel von krebsgemeinschaft.de}

Wichtige Designelemente für eine VC für Patienten sind die Informationsaufbereitung, die Funktionalitäten, Nutzerfreundlichkeit (Usability) und Accessibility (i. S. v. Barrierefreiheit) des Angebots sowie der Einsatz vertrauensunterstützender Komponenten [Leim04], auf die jeweils im Folgenden eingegangen wird.

\subsection{Informationsdienste}

Der Schwerpunkt im Bereich Informationsdienste liegt in der großen Menge an redaktionell aufbereitetem und qualitätsgesichertem Inhalt zum Thema Brustkrebs. Die Erarbeitung einer Struktur für diesen komplexen Inhalt ist eine der zentralen Herausforderungen neben der laienverständlichen Aufbereitung.

Im Rahmen einer iterativen Entwicklung und dem Durchlaufen mehrerer Runden an Expertengesprächen wurde die folgende Kategorisierung erarbeitet und in der Rangfolge der erwarteten Relevanz für die Nutzer gereiht: a) Krebs behandeln (Therapie, Nachsorge); b) Krebs erkennen (Früherkennung, Diagnosemethoden); c) Krebs erforschen (Studien, Zahlen \& Daten); d) Leben mit Krebs (Sport \& Ernährung, Familie \& Sexualität) und e) Erfahrungen mit Krebs (Betroffene berichten). 
Die Aufteilung der Oberkategorien in kleinere Untereinheiten soll dem Nutzer helfen, die dargebotenen Informationen einfach strukturiert schneller wahrzunehmen und besser kognitiv zu verarbeiten.

\subsection{Funktionalitäten}

Im Bereich der Funktionalitäten werden die Dienste Diskussionsforum, Fragen an Experten, Kontaktsuche und Chat angeboten. Darüber hinaus werden über d ie personalisierte Startseite der Mitglieder ,,persönliche“ Interaktionsdienste wie ein internes Mail-System oder das eigene Gästebuch, oder aber Informationsdienste wie Freundeslisten und Awareness-Funktionen wie „Freunde online“, „Nutzer im Chat" oder „Mitglieder in der krebsgemeinschaft.de“ bereitgestellt (vgl. Abbildung 1).

Das Diskussionsforum ermöglicht den asynchronen Austausch der Mitglieder untereinander. Damit unterstützt es neben der Kommunikation auch den Prozess des Kennenlernens der Mitglieder untereinander und fördert somit den Aufbau und die Pflege einer Gemeinschaft.

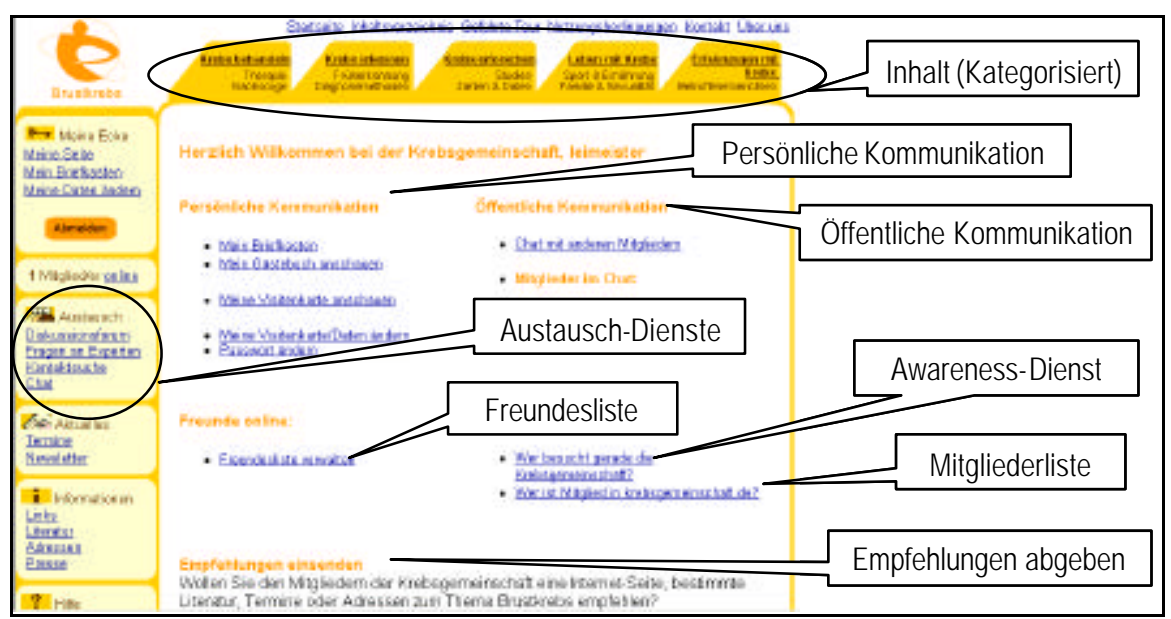

Abbildung 1: Informations- und Interaktionsdienste sowie graphische Aufbereitung der persönlichen Startseite der Mitglieder von www.krebsgemeinschaft.de (Quelle: [Leim04, S. 201]).

Der Dienst „Fragen an Experten“ ist eine abgewandelte Form eines Diskussionsforums, in dem die Nutzer Fragen stellen und nur die Experten antworten können. Darüber hinaus sind die Dauer einer Fragerunde und auch das Thema vorgegeben. Auf diese Weise wird der Aufwand für die beantwortenden Experten reduziert und besser planbar. Nachfragen zu Antworten etc. können von den Nutzern ohne Zeitdruck in aller Ruhe in das Diskussionsforum gestellt werden. 
Die Kontaktsuche hat zum Ziel, den Mitgliedern von krebsgemeinschaft.de eine Unterstützung bei der Suche nach Menschen in ähnlichen Lebenslagen und/oder mit ähnlichen Interessen zu bieten. Es soll die einfache und unkomplizierte Möglichkeit der Kontaktaufnahme mit anderen Mitgliedern geschaffen werden, um so den persönlichen Erfahrungs- und Meinungsaustausch sowie die Bildung eines Gemeinschafts gefühls zu fördern.

Der Chat bietet den Nutzern die Möglichkeit, miteinander zeitgleich in Kontakt zu treten, er ist eine synchrone Echtzeitkommunikation, die mindestens zwei Teilnehmer erfordert. Um in der virtuellen Community die Wahrscheinlichkeit, einander zu treffen zu erhöhen, wurden bspw. im betrachteten Fall feste Zeiten für den Chat vorgegeben [Brun ${ }^{+} 00$, S. 137].

\subsection{Nutzerfreundlichkeit (Usability)}

Angebote im Internet zeichnen sich dadurch aus, dass potentielle Nutzer innerhalb weniger Sekunden über deren Nutzung entscheiden [WaKr99, S. 1]. Neben der Bedarfsgerechtigkeit der angebotenen Inhalte kommt insbesondere der Nutzerfreundlichkeit besondere Bedeutung zu. Daher spielen Aspekte wie Layout, Farb-, Graphik- und Schrift gestaltung sowie Ergonomie eine wichtige Rolle. Diese Aspekte werden in der Literatur meist unter dem Schlagwort der Usability behandelt. Usability ist immer kontext - und verwendungsspezifisch, daher kann es keine detaillierten, allgemeingültigen Regeln hierfür geben. Usability und Gesamtanmutung haben außerdem entscheidenden Einfluss darauf, dass Websites Glaubwürdigkeit und Vertrauen ausstrahlen [Fogg $\left.{ }^{+} 02\right]$. Aus der Vielzahl unterschiedlicher Usability-Prinzipien lassen sich folgende Metaprinzipien unterscheiden:

Aufgabenangemessenheit: ein Dialog mit einem System gilt dann als angemessen, wenn er die Erledigung der Arbeitsaufgabe des Benutzers unterstützt, ohne ihn durch Eigenschaften des Dialogsystems unnötig zu belasten.

Erwartungskonformität: ein System verhält sich dann erwartungskonform, wenn jedes Element/jeder Teil dem Benutzer das Design und den Inhalt präsentiert, den dieser an dieser Stelle erwartet.

Konsistenz: ein einheitliches Erscheinungsbild eines Systems für ėnen Kontext wird als "Konsistenz" bezeichnet. Konsistenz ist für die Usability ausschlaggebend, weil der Benutzer auf bereits Gelerntes zurückgreifen kann und sich nicht neu anpassen bzw. neu lernen muss. Konsistenz ist eines der wichtigsten Usability-Prinzipien [Mayh92] und führt zu Usability [Spo101; Nie102].

Visibility: Gute Mensch-Maschine-Schnittstellen haben gut sichtbare Bedienungselemente. Jedes Bedienelement (Knopf, Schaltfläche, etc.) kontrolliert hierbei idealerweise nur eine Funktion. 


\subsection{Barrierefreiheit (Accessibility)}

Wenn das Internet eine Weitergabe von Information als Text sicherstellt, kann es bei physischen Behinderungen dazu beitragen, diese zu überwinden. Dabei kann die Behinderung eines Sinnesorgans durch die Nutzung eines anderen ersetzt werden [WEBf03a]. Diese Thematik ist für eine Zielgruppe wie Krebspatienten besonders wichtig, da sie je nach Behandlungsstadium mit zeitweise eingeschränkter Mikromobilität und körperlichen Behinderungen umzugehen hat. Auf Internetseiten entstehen in Abhängigkeit von den verwendeten Gestaltungsmitteln verschiedene Barrieren für unterschiedliche Arten von Behinderungen.

Seit dem 1. Mai 2002 gilt das Behindertengleichstellungsgesetz (BGG), das die Benachteiligung von behinderten Menschen zu beseitigen und zu verhindern anstrebt. §11 BGG schreibt vor, dass Träger öffentlicher Ge walt ihre Internetauftritte und -angebote sowie die von ihnen zur Verfügung gestellten graphischen Programmoberflächen, die mit Mitteln der Informationstechnik dargestellt werden, so gestalten, dass sie von behinderten Menschen grundsätzlich unein geschränkt genutzt werden können. Eine barrierefreie Internetseite ist mit den derzeit zur Verfügung stehenden Hilfsmitteln problemlos lesbar und navigierbar. Um dies zu erre ichen, wurden von unterschiedlichen Anbietern Ge staltungsempfehlungen erarbeitet, die im Ergebnis zu barrierefreien Internetseiten führen sollen (vgl. hierzu bspw. [WEBf03b] oder auch vertiefend [Leim04, S. 183ff]).

\subsection{Vertrauensunterstiitzende Komponenten}

Neben einer benutzerfreundlichen Oberfläche und nutzenstiftenden Diensten der Community-Plattform ist die Vertrauenswürdigkeit des Angebotes ein zentraler Erfolgsfaktor. Entscheidende Stellschrauben für die Unterstützung von Vertrauen sind das Betreiberkonzept (wer betreibt es mit welchem Hintergrund und welcher Motivation), ein Rechte- und Rollenkonzept (welche Aufgaben und Rollen existieren bei dem Angebot, wer nimmt sie wahr und welche Rechte hat jeder Akteur), ein Anonymitätskonzept (Möglichkeit jedes Nutzers, selbst darüber zu entscheiden, welche und wie viele seiner persönlichen Daten für andere Nutzer sichtbar werden) sowie die Verwendung von Qualitätssiegeln nebst entsprechenden Routinen für die Qualitätssicherung der eigenen Inhalte und Angebote. Hierzu wurden im Falle von krebsgemeinschaft.de die Richtlinien und Transparenzkriterien von afgis (Aktionsforum Gesundheitsinformationssysteme e.V., http://www.afgis.de) verwandt. Für detailliertere Informationen zur Ausgestaltung der vertrauensunterstützenden Komponenten siehe auch [Ebne ${ }^{+}$03; Leim04]. 


\section{Akzeptanz und Nutzen von krebsgemeinschaft.de}

\subsection{Evaluationskriterien und Evaluationsmethoden}

Um Aussagen über die Wirksamkeit der im Rahmen der Pilotierung von krebsgemeinschaft.de durchgeführten Maßnahmen machen zu können, sind zunächst Bezugsgrößen für den Erfolg sowohl der jeweiligen Maßnahmen als auch der Cesamtheit aller Maßnahmen (in dem hier betrachteten Fall demzufolge der gesamten Virtuellen Community) zu bestimmen. Eine Erfolgsmessung bzw. eine Ursache-Wirkungskette ist bei vielen Einzelmaßnahmen jedoch nicht möglich, da sie nicht losgelöst von anderen Einflussgrößen auf den Nutzer wirken und ihre Wirkung daher nicht isolierbar ist. Oftmals ist sogar nur die Summe mehrerer Maßnahmen und Einflussgrößen als Gesamtheit erfassbar und bewertbar. Abbildung 1 verdeutlicht die wichtigsten zuvor dargestellten Designmaßnahmen und ihre Wirkung (als Gesamtheit) auf den Erfolg bzw. die Akzeptanz und den Nutzen von krebsgemeinschaft.de

\begin{tabular}{||c||} 
Informationsdienste \\
Usability (Farben \& Formen, Navigation, etc.) \\
Funktionalitäten \\
Barrierefreiheit (Accessibility) \\
$\begin{array}{c}\text { Vertrauensunterstützende Komponenten } \\
\text { (Betreiberkonzept, Rollen- und Rechtekonzept, } \\
\text { Anonymitätskonzept) }\end{array}$
\end{tabular} \mid $\begin{gathered}\text { Akzeptanz } \\
\text { \&utzen } \\
\text { von } \\
\text { krebsgemein- } \\
\text { schaft.de }\end{gathered}$

Abbildung 1: Intendierter Wirkungszusammenhang zwischen Design- \& Einführungs maßnahmen und Nutzung/Nutzen von krebsgemeinschaft.de

Spezifischere Indikatoren für den Erfolg Virtueller Gemeinschaften lassen sich auf unterschiedlichen Ebenen finden, wenngleich es an systematischen und operationalisierbaren Vorschlägen mangelt. Für die Evaluation bieten sich sowohl Techniken aus der „klassischen“ Sozialforschung als auch aus der Online-Forschung an. Hierzu werden im Folgenden Log-File-Analysen, Online-Befragungen, Dokumentenanalysen und Beobachtungen verwandt. Im Folgenden wird auf die Anwendung unterschiedlicher Methoden zur Evaluation der VC krebsgemeinschaft.de eingegangen. Alle Angaben beziehen sich (sofern keine anderen Angaben gemacht werden) auf die Zeitspanne vom 19.08.2002 (Online-Schaltung der VC) bis zum 22.05.2003. Zunächst wird auf die (quantitative) Analyse der ServerLog-Files eingegangen, danach auf eine Online-Umfrage unter den Nutzern von krebsgemeinschaft.de und abschließend werden ausgewählte Dokumentenanalysen und Beobachtungen durchgeführt und analysiert. 


\subsection{Empirische Ergebnisse}

\subsubsection{Kennzahlen zur Bewertung der interaktiven Community-Elemente}

Den Kern einer VC bildet die Interaktion zwischen den Mitgliedern, die entweder in Chats oder Diskussionsforen stattfindet. Zur Bewertung der „Member-tomember interaction“"werden folgende Kriterien herangezogen (in Anlehnung an [Coth00, S. 18]).

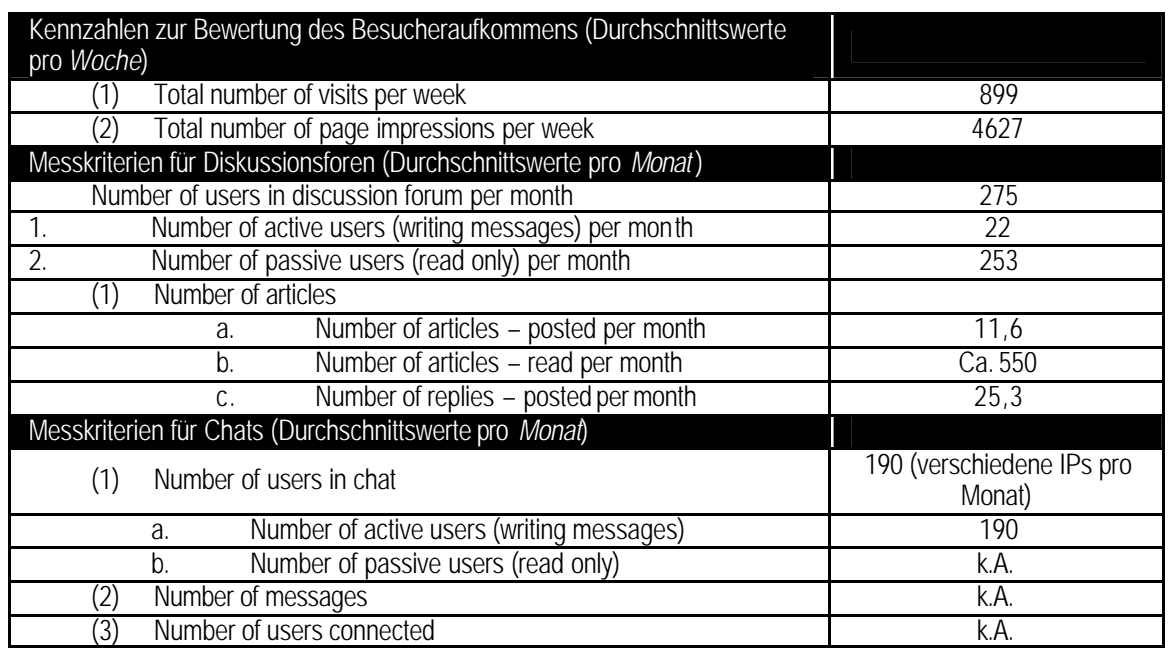

Tabelle 1: Kennzahlen von krebsgemeinschaft.de für die Periode 08/2002-05/2003

Weiterhin muss beachtet werden, dass die Log-File-Analyse und damit die rein quantitativen Werte zur Beurteilung einer VC nicht hinreichend ist. So kann bspw. ein kleiner Kreis von aktiven Nutzern (wie dieser sich auch bei krebsgemeinschaft.de herausgebildet hat) viel stärker zum „Leben“ einer Community beitragen als ein großer Kreis passiver Nutzer, sog. „Lurker“ [NoPr00]. Aus diesem Grund werden im Anschluss an die Log-File-Analysen auch qualitative Analysen von krebsgemeinschaft.de durchgeführt, u. a. in Form von Befragungen, Beobachtungen und Inhaltsanalysen und die jeweiligen Ergebnisse i. S. einer Methodentriangulation miteinander verglichen.

\subsubsection{Nutzung der einzelnen Bereiche und Dienste}

Folgend wird auf die Nutzung der einzelnen Bereiche und Dienste des Angebots krebsgemeinschaft.de eingegangen. Zunächst wird die Nutzung der redaktionell aufbereiteten Inhalte analysiert und anschließend auf die Nutzung der Interaktionsdienste (Chat, Forum, Fragen an Experten, Kontaktsuche) eingegangen. 
Nutzung des redaktionellen Inhalts - Inhaltskategorien der obersten Ebene die graphische Darstellung und Positionierung der Inhalte auf der Website ist in Abbildung 1 dargestellt. Die Inhaltskategorien und ihre Anordnung sind analog zur Wichtigkeit/Häufigkeit der Themen für Krebspatienten bei anderen Medien und Angeboten erfolgt. In abfallender Rangfolge (analog zur erwarteten abnehmenden Nutzungshäufigkeit) sind die Content-Reiter-Titelseiten (obere Inhaltsnavigation von krebsgemeinschaft.de) mit den Kategorien ,Krebs behandeln', ,Krebs erkennen', ,Krebs erforschen', ,Leben mit Krebs' und ,Erfahrungen mit Krebs' von links nach rechts angeordnet worden.

Für alle Inhaltskategorien (Content-Reiter) ist eine ähnliche Entwicklung in den Nutzungszahlen zu beobachten. Für alle Content-Reiter fallen die Nutzungszahlen nach den ersten Wochen ab, pendeln sich dann aber auf einem mehr oder minder stabilen Niveau ein. Relativ hohe Werte auch in den letzten Monaten weisen vor allem die erste Kategorie „Krebs behandeln“ und die Kategorie „Leben mit Krebs“ auf (entgegen der ursprünglichen Annahme, dass Leben mit Krebs auf Rang 4 der Inhaltskategorien wäre). Diese Themen sind demzufolge für die Besucher von krebsgemeinschaft.de von besonders hohem Interesse. Da sich die redaktionellen Inhalte über einen längeren Zeitraum nicht verändert haben, ist anzunehmen, dass Besucher, die mehrmals die Seiten besucht haben, ihren Informationsbedarf hieraus bereits gedeckt haben und nicht mehr oder nur vereinzelt zu den Seiten zurückgekehrt sind. Außerdem kamen in der Zwischenzeit auch relativ wenig ,neue“ Mitglieder mit in die VC. Hierdurch lassen sich möglicherweise insgesamt fallende Nutzungszahlen in diesem Bereich erklären, ein erster möglicher Hinweis auf einen nutzerindividuellen Informationsbedarfszyklus, der u. U. auch abhängig vom Krankheits stadium bzw. von der Zeitspanne seit der Diagnose abhängig sein könnte.

Austauschseiten/Interaktionsseiten - die Analyse der Entwicklung der Nutzung der einzelnen Austausch- und Interaktionsseiten zeigt deutlich, dass vor allem die Kontaktsuche sehr wenig genutzt wird. Dies mag einerseits daran liegen, dass sich nach einiger Zeit bereits feste Gruppen innerhalb von krebsgemeinschaft.de gebildet haben, die miteinander interagie ren und daher nicht auf die Kontaktsuche angewiesen sind, andererseits kann es an der u. U. nicht zufrieden stellenden Umsetzung der Kontaktsuche liegen. Im Rahmen der Umfragen ist zumindest von mehreren Nutzern erwähnt worden, dass die Kontaktsuche nicht wunschgemäß funktioniere bzw. zu lange dauere.

Diskussionsforum - das Diskussionsforum wird relativ rege genutzt. Insgesamt wurden über den kompletten Evaluationszeitraum hinweg 367 Einträge im Diskussionsforum eingestellt. Hiervon waren 114 Beiträge neue Threads und 253 Beiträge Replies auf diese Threads. Dies bedeutet, dass es im Schnitt auf jede Anfrage mehr als 2 Antworten gab, wobei die Zahl der Antworten zwischen 0 (häufig war auch keine Antwort erwartet, z. B. wenn es sich um reine Hinweise auf Vorträge, Expertenrunden o. ä. handelte) und 9 Antworten auf eine Anfrage schwank- 
te. Meist waren die Antworten konkret auf die Anfrage bezogen, nur selten entwickelte sich eine Diskussion, die gänzlich vom Ursprungsthema abwich.

Um festzustellen, welche Themen im Diskussionsforum besprochen wurden, wird nachfolgend eine Inhaltsanalyse der Threads und Replies durchgeführt. Grundsätzlich können zwei große Themenblöcke identifiziert werden: Einträge, die eher rationale/sachliche Informationsbedürfnisse aufzeigen, und emotionale Einträge, die einen Bedarf nach zwischenmenschlicher Unterstützung, emotionaler Integration in eine Gemeinschaft und den Wunsch nach Empathie zeigen.

Häufig waren diese beiden Themenblöcke nicht trennscharf zu unterscheiden, da auch Anfragen nach Informationen oftmals sehr emotional geprägt waren, z. B. wenn es um Haarverlust bei der Chemotherapie oder um künstlichen Brustaufbau nach Amputation der Brust ging.

Insgesamt zeigt sich, dass das Diskussionsforum vor allem verwendet wird, um Erfahrungen mit anderen zu teilen, bzw. aus den Erfahrungen anderer Mitglieder zu lernen. Und obwohl bei dieser Kategorisierung der Großteil der Beiträge unter eher „rationale“ Kategorien zu subsumieren ist, bleibt zu bemerken, dass viele, auch sehr informativ geprägte Beiträge einen emotionalen Aspekt enthalten. Auffallend ist auch, dass sehr persönliche Beiträge ins Forum gestellt werden und die Mitglieder sehr offen über ihre Gefühle und persönlichen Erfahrungen sprechen. Allerdings muss auch bemerkt werden, dass insbesondere in den ersten Monaten ein Großteil der Interaktion durch einige wenige Mitglieder entstanden ist, die sehr stark in der VC engagiert sind.

Visitenkarten und Gästebücher - Das Anonymitätskonzept von krebsgemeinschaft.de gibt dem Nutzer die Möglichkeit, selbst darüber zu entscheiden, welche seiner Daten er anderen Mitgliedern der Gemeinschaft auf seiner Visitenkarte ze igen möchte. Dabei kann zwischen vier verschiedenen Anonymitätsgraden gewählt werden: Nichts anzeigen (andere Mitglieder bekommen die Anzeige: „Der Benutzer möchte seine Daten nicht anzeigen!"), anonymisiert (angezeigt werden: Benutzername, Bundesland, Nutzerstatus, Verbindung zur Krankheit, Diagnosedatum, Krebsart, Krebsstadium, Therapieform, Freizeitaktivitäten, Interessen (sonstiges)), alle anonymisiert, Freunde alles (Mitgliedern werden angezeigt: Benutzername, Bundesland, Nutzerstatus, Interessen, Verbindung zur Krankheit, Diagnosedatum, Krebsart, Krebsstadium, Therapieform, Freizeit aktivitäten, Interessen (sonstiges), Freunden werden alle eingegebenen Daten angezeigt) und alles anzeigen (Angezeigt werden: Alle eingegebenen Daten). Die Visitenkarte ist über dem jeweiligen Gästebuch des Mitglieds angebracht.

Im Mai 2003 gab es 634 Gästebücher auf krebsgemeinschaft.de. 273 (43,1\%) Mitglieder hatten dabei den Anonymitätsgrad ,nichts anzeigen“ gewählt, 272 (42,9\%) den Anonymitäts grad ,,anonymisiert“", $9(1,4 \%)$,alle anonymisiert, Freunde alles“ und $80(12,6 \%)$ ließen sämtliche Daten allen anderen Mitgliedern der VC anzeigen. Betrachtet man nun in Abhängigkeit vom Anonymitätsgrad des Gästebuches die Zahl der Einträge anderer Mitglieder in das jeweilige Gästebuch, so 
werden klare Unterschiede deutlich: Während in die 273 Gästebücher mit dem Anonymitätsgrad „,nichts anzeigen“ insgesamt nur 30 Einträge gemacht wurden, enthielten die 272 Gästebücher mit dem Anonymitätsgrad ,,anonymisiert“ immerhin schon 217 Einträge. In die 80 Gästebücher, die für alle anderen Mitglieder offen waren wurden 383 Einträge gemacht. In die 9 Gästebücher mit dem Anonymitätsgrad ,,alle anonymisiert, Freunde alles“ wurden 3 Einträge gemacht.

Dies verdeutlicht, dass Offenheit eine wichtige Grundvoraussetzung für das Funktionieren der VC ist. Je mehr Daten für andere einsehbar sind, desto mehr Einträge werden ins Gästebuch gemacht. Je mehr Offenheit und Vertrauen (hier durch das Anzeigen persönlicher Daten) anderen Nutzern also entgegengebracht wird, des to mehr wird auch zurückgegeben (hier in Form von Einträgen ins Gästebuch). Dies entspricht auch dem aus der Literatur als „Reciprocity“ bezeichneten Prinzip der Wechselseitigkeit [Pree99; Pree00; PrMa03]. Hiernach ist das Geben von eigenen Informationen an die Gemeinschaft der Katalysator für das Erhalten von Informationen und Reaktionen durch die Ge meinschaft bzw. durch andere Mitglieder.

Wichtig ist aber anzumerken, dass die Verteilung der Gästebucheinträge asymmetrisch ist. Auf der einen Seite enthält ein Großteil der Gästebücher überhaupt keine Einträge, auf der anderen Seite haben einige sehr aktive Mitglieder in krebsgemeinschaft.de Gästebücher mit jeweils mehr als 40 Beiträgen.

Dies deutet - wie auch die Tatsache, dass viele der Einträge im Diskussionsforum von einigen wenigen Nutzern gepostet wurden - darauf hin, dass innerhalb von krebsgemeinschaft.de eine kleine, sehr aktive Gemeinschaft von Nutzern entstanden ist, die sich sehr rege austauscht. Diese Gruppe trägt stark zum Leben innerhalb der VC bei und krebsgemeinschaft.de scheint eine wichtige Rolle für diese Mitglieder zu spielen. Das Verhältnis dieser aktiven Nutzer zueinander scheint sehr eng zu sein und der Austausch geht oftmals auch über die Commu nityPlattform hinaus. Diese Mitglieder nehmen sehr stark an der Situation und an den Problemen der anderen aktiven Mitglieder Anteil und versuchen soweit möglich auch gegenseitig für einander da zu sein.

\subsubsection{Akzeptanz und Nutzen von krebsgemeinschaft.de}

Anhand quantitativer Analysen lassen sich nur bedingt Erkenntnisse über die individuelle Nutzung und vor allem auch die Nutzbarkeit (Usability) der Plattform krebsgemeinschaft.de ziehen. Noch schwieriger ist es, Erkenntnisse über den aus der Nutzung gezogenen Nutzen der Teilnehmer zu gewinnen. Unter Nutzung wird folgend die (auf Selbstauskunft beruhende) Häufigkeit der Nutzung der einzelnen Angebote innerhalb von krebsgemeinschaft.de verstanden. Hierzu wurde analog zur Konzeption der Plattform zwischen Informations- und Kommunikations- (Interaktions-) diensten unterschieden. $\mathrm{Zu}$ beiden Bereichen wurden sowohl geschlossene als auch offene Fragen gestellt. Unter Nutzen wird folgend der subjektive Eindruck der Teilnehmer verstanden, welche persönlichen Vorteile sie aus 
der Nutzung von krebsgemeinschaft.de ziehen. Hierzu wurden ebenfalls sowohl offene als auch geschlossene Fragen gestellt.

Gesamtbeurteilung - insgesamt beurteilten die Teilnehmer der Umfrage den Aufbau von krebsgemeinschaft.de positiv (vgl. Abbildung 2). Dies überrascht nicht, da es sich bei den Teilnehmern überwiegend um Personen handelt, die das Angebot stark nutzen. Eine freiwillige wiederholte Nutzung kann für sich genommen schon als positive Beurteilung des Angebots gewertet werden.

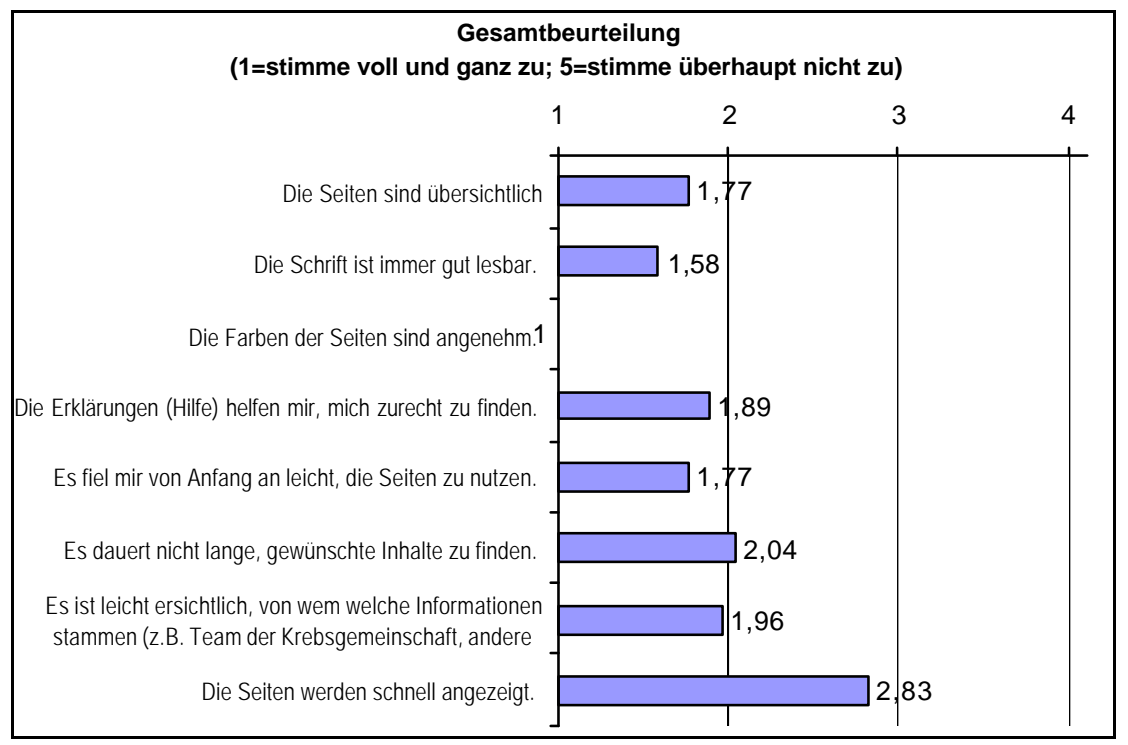

Abbildung 2: Gesamtbeurteilung von krebsgemeinschaft.de $(\mathrm{n}=27)$

Im Schnitt konnten die Nutzer zustimmen, dass die Seiten übersichtlich und gut lesbar sind, dass die Bedienung leicht sei und gewünschte Inhalte schnell zu finden seien. Besonders positiv wurde die farbliche Gestaltung beurteilt. Auch die Quelle der Informationen und Beiträge scheint den Teilnehmern klar erkenntlich. Als schlechter beurteilt wurden die Ladegeschwindigkeit der Seiten sowie die technische Performanz der Plattform insgesamt.

Zusammenhang zwischen der Nutzung unterschiedlicher Angebote von krebsgemeinschaft.de und der Mitgliedschaftsdauer -die Analyse ergibt, dass es einen erkennbaren Zusammenhang zwischen der Mitgliedschaftsdauer und der Nutzung des Informationsangebotes gibt. 55,5\% derjenigen, die weniger als 6 Monate Mitglied sind, nutzen das Informationsangebot jedes Mal/meistens. Demgegenüber nutzen nur $40 \%$ der Mitglieder, die länger als 6 Monate bei krebsgemeinschaft.de sind, das Angebot jedes Mal / meistens. Dies legt die Schlussfolgerung nahe, dass die Bedeutung des Informationsangebotes mit andauernder Mitgliedschaft abnimmt. 
Ein noch deutlicherer Zusammenhang besteht zwischen der Mitgliedschaftsdauer und dem Interaktionsangebot. Die Nutzung des Interaktionsangebotes nimmt mit andauernder Mitgliedschaftsdauer zu. 38,5 \% derjenigen, die länger als 6 Monate bei krebsgemeinschaft.de sind, nutzen das Interaktionsangebot jedes Mal/meistens, wohingegen keiner der Nutzer, die weniger als 6 Monate Mitglied sind, dies angab.

Diese Ergebnisse sind Indizien für einen individuellen Mitgliederzyklus, der sich in unterschiedlichen Nutzungsphasen des Systems manifestiert. Es ist bei einem neu mit der Krankheit konfrontierten neuen Nutzer von krebsgemeinschaft.de davon auszugehen, dass am Anfang ein sehr hoher Bedarf nach „harten Fakten“ zu der Krankheit besteht. Im Laufe der Zeit eignet sich der Betroffene oftmals weit reichendes Wissen selbst an und greift daher nur noch seltener auf das Informationsangebot zurück. Entgegengesetzt verhält es sich mit dem Interaktionsverhalten. Dieses steigt oftmals eher mit längerer Zugehörigkeit zu einer Gemeinschaft, da sich engere soziale Beziehungen ausbilden, welche wiederum $\mathrm{zu}$ intensiverer $\mathrm{In}$ teraktion führen.

Nutzen - die Analyse einer direkten Abfrage des empfundenen Nutzens von krebsgemeinschaft.de durch die Mitglieder zeigt - ähnlich der Ergebnisse der Analyse der Beiträge im Diskussionsforum - zwei Hauptgründe für die Nutzung von krebsgemeinschaft.de: Zum einen liefert das Angebot Betroffenen und Angehörigen fachliche Informationen zum Thema Krebs, zum anderen - und dies scheint der wichtigere Grund von beiden zu sein - kann Kontakt zu anderen Personen, die in einer ähnlichen Situation sind, aufgebaut werden und es können sich soziale Beziehungen und Empathie entfalten. Weiterhin können sich die Mitglieder emotional in eine Gemeinschaft integrieren und sich gegenseitig Halt und emotionale Unterstützung geben. Dies geht sogar soweit, dass in Beiträgen genannt wird, dass sich Mitglieder von krebsgemeinschaft.de schon vermehrt "real" getroffen haben.

Folgende Antworten auf die Frage ,Bitte beschreiben Sie kurz, wie sich die Mitgliedschaft bei krebsgemeinschaft.de auf ihre Situation auswirkt/ausgewirkt hat.“, verdeutlichen die Be deutung des Angebots für die Mitglieder:

„Der Austausch mit Betroffenen ist sehr wohltuend. Gerade nach der Krankheit gehen alle davon aus (Familie, Beruf), dass das Leben wieder so ist wie vorher. Dem ist nicht so !!! Natürlich will man nicht ständig über das (schlechte) Befinden sprechen und irgendwie will das ja auf Dauer auch niemand hören. Deshalb ist der Austausch mit Betroffenen wichtig, es wird Mut gemacht und es hilft zu wissen, dass man in dieser Situation nicht alleine auf der Welt ist. Es ist auch sehr beeindruckend zu lesen, wie manche Frauen mit ihrem Schicksal umgehen. Das weckt den Kämpfergeist und hilft, den Heilungsprozess oder auch Rückschläge besser zu verarbeiten. “

Bei der Konzeption und beim Aufbau von krebsgemeinschaft.de wurden vertrauensfördernde Komponenten konzipiert und in die VC integriert. Der Prozess der 
Vertrauensbildung sollte durch diese Komponenten unterstützt werden, um so zum erfolgreichen Aufbau und Betrieb der Gemeinschaft beizutragen. Die vorliegenden Ergebnisse einer Online-Umfrage, die zum Ziel hatte, das Vertrauen in die VC krebsgemeinschaft.de zu messen, scheint den Erfolg dieser vertrauensbildenden Komponenten zu bestätigen. Sowohl das Vertrauen in die Betreiber von krebsgemeinschaft.de, hier vor allem in deren fachliche Kompetenz, als auch das Vertrauen in die anderen Mitglieder der VC, hier vor allem in das wahrgenommene Wohlwollen, scheinen hoch zu sein. Auch die Handlungsweisen der befragten Mitglieder entsprechen diesem Frgebnis: Sie sind sowohl bereit, Informationen, die innerhalb der Community zur Verfügung stehen, zu nutzen, als auch selbst persönliches Erfahrungen und eigenes Wissen zur Verfügung zu stellen. Damit zeigen sie, dass sie sowohl darauf vertrauen, dass persönliche Informa tionen nicht missbraucht werden, als auch darauf, dass bereits in der Gemeinschaft vorhandenen Infomationen und Daten als richtig erachtet werden. Darüber hinaus konnte nachgewiesen werden, dass die Befragten zum Großteil sogar entsprechend dieser Informationen handeln (für weitergehende Informationen zur Entwicklung und Evaluation vertrauensunterstützender Komponenten s. [Leim04]).

\section{Fazit}

Die Evaluation von krebsgemeinschaft.de hat viele unterschiedliche Erkenntnisse zu Tage gefördert. Es handelt sich im betrachteten Fall von krebsgemeinschaft.de um ein erfolgreiches Angebot. Die Mitgliederzahlen steigen kontinuierlich, es gibt eine konstante aktive Kerngemeinschaft sowie eine „erweiterte“ Gemein schaft mit passiven Mitgliedern (Lurkern). Innerhalb der aktiven Nutzerschaft funktioniert das Prinzip der Wechselseitigkeit der Mitglieder bzw. des aktiven Austauschs von Informationen, Empathie und Unterstützung sehr rege. Die Interaktion ist oftmals von großer Empathie geprägt und es herrscht relativ hohes Vertrauen in die Betreiber der VC ebenso wie in die anderen Mitglieder. Die Konzeption vertrauensunterstützender Komponenten hat hiernach zum ge wünschten Erfolg geführt.

Die Anzahl an Verstößen gegen die Regeln der Gemeinschaft oder Vorfällen, die als grob oder unhöflich einzustufen sind, ist ausgesprochen gering. Die aktiven Mitglieder zeigen sich darüber hinaus sehr zufrieden mit der sozialen Interaktion in der VC und die Tatsache, dass fast alle Mitglieder der „ersten Stunde“ immer noch aktiv in der Gemeinschaft sind, spricht für eine relativ hohe Treue und Bindung an krebsgemeinschaft.de.

Bezogen auf die Bedarfsgerechtigkeit der Community-Plattform zeigen sich die Mitglieder größtenteils zufrieden, wenngleich einzelne Funktionalitäten unterschiedlich stark wertgeschätzt werden. Außerdem zeigt sich, dass das Design (bspw. bei Oberflächenentwicklung und Funktionalitäten) weitgehend als nutzergerecht und gut eingeschätzt wird. Jedoch zeigen die Nutzerbefragungen auch, dass manche Eigenschaften der VC vielen Nutzern nicht oder nicht hinreichend 
genau bekannt sind und daher nicht verwandt werden (bspw. das Anonymitätskonzept und die Kontaktsuche). Außerdem zeigt sich auch, dass technische Zuverlässigkeit der Plattform den Nutzern wichtiger ist als ausgefeilte Dienste bzw. Funktionalitäten. Außerdem wird deutlich, dass Technik nicht der limitierende Faktor ist, sondern dass oftmals die organisatorische Umsetzung bspw. eines Dienstes viel ausschlaggebender für den Erfolg ist. Deutlich wird das an der sehr unterschiedlichen Nutzung des Dienstes „Fragen an Experten“, bei dem durch schnelle Antwortzeiten der Experten und qualitativ hochwertige, vor allem aber auch laienverständliche Antworten der Nutzen und damit auch die Nutzung des Dienstes massiv gesteigert werden kann. Ebenso zeigt die Evaluation die zentrale Rolle qualitativ hochwertigen Inhalts vor allem als Initialangebot an potentielle Nutzer. Ein weiterer kritischer Erfolgsfaktor beim Aufbau der VC ist die Rolle des Community-Managements, das bei noch nicht Erreichen der kritischen Masse an Nutzern für Aktivität und Attraktivität der entstehenden VC sorgen muss.

Die Evaluation zeigt weiterhin, dass es eine Verschiebung der Informations- und Interaktionsbedarfe der Nutzer in Abhängigkeit ihrer Zugehörigkeitsdauer bei der VC gibt. So erscheinen der Wunsch nach Interaktion und vor allem auch die Wertschätzung derselben bei aktiven Mitgliedern über die Zeit hinweg zu steigen.

Konsequenzen für die Forschung - Zunächst sind die im Rahmen dieser explorativen Untersuchung gewonnenen Ergebnisse mit VCs aus anderen Lebensbereichen/anderen Arten von VCs zu vergleichen und die Belastbarkeit der Ergebnisse $\mathrm{zu}$ erhöhen. Es kann auf Basis dieser Arbeit ein quantitativ überprüfbares Modell erstellt werden und die Theorieentwicklung zu Virtuellen Gemeinschaften vorangetrieben werden.

Weiterhin ergeben sich Chancen und Herausforderungen für VCs durch neue technische Möglichkeiten. So sind ubiquitäre Zugänge zu VCs durch mobile Endgeräte ebenso denkbar wie neue (mobile bzw. kontextsensitive) Dienste für VCs (bspw. ein mobiler Notrufdienst für Krebspatienten mit sofortiger Lokalisierung oder ein mobiler Medikamentenreminder-Dienst, deren Rolle und Nutzen für VCs für Patienten noch nicht abgeschätzt werden können).

Des Weiteren besteht Forschungsbedarf zu Rolle und Aufgaben des CommunityManagements. Es fehlen weitgehend gehaltvolle Arbeiten zu sinnvollen Unterstützungstools (insbesondere bei einer Vielzahl betriebener VCs) für das effiziente und effektive Management.

Auch ist die Analyse der sozialen Interaktionen in VCs und die Auswirkungen auf bspw. das soziale Netzwerk von Teilnehmern einer VC weitgehend unerforscht. Ebenso vielversprechend erscheint die Untersuchung des genauen Nutzens insbesondere gesundheitsbezogener VCs, bspw. in Form der Messung des Einflusses auf die empfundene Lebensqualität und damit mittelbar auf Kriterien wie Lebenserwartung und Behandlungskosten. 
Zusammengefasst ist das Ergebnis dieser Evaluation ein empiris cher Nachweis, dass die bei krebsgemeinschaft.de gewählten Ansätze zur bedarfsgerechten Entwicklung, Einführung und Betrieb einer Virtuellen Gemeinschaft für Krebspatienten im deutschen Gesundheitswesen erfolgreich waren. Sie vermittelt ein reiches Verständnis von interdependenten Einflussgrößen auf Virtuelle Gemeinschaften im Allgemeinen und liefert einen inhaltlichen Beitrag zum Verständnis des Community-Buildings für Patienten.

\section{Literatur}

[Arms01] Armstrong, L.: It's Not About the Bike: My Journey Back to Life. Yellow Jersey Press, London 2001.

[BiDe96] Bilodeau, B. A.; Degner, L. F.: Information needs, sources of information, and decisional roles in women with breast cancer. In: Oncology Nursing Forum, Vol. 23 (1996), Nr. 4, S. 691-696.

$\left[\right.$ Brun $\left.^{+} 00\right]$ Brunold, J.; Merz, H.; Wagner, J.: www.cyber-communities.de - Virtual Communities: Strategie, Umsetzung, Erfolgsfaktoren. mi, Verlag Moderne Industrie, Landsberg/Lech 2000.

[Coth00] Cothrel, J.: Measuring the success of an online community. In: Strategy \& Leadership, Vol. 28 (2000), Nr. 28.02.2000, S. S. 17-21.

[Daum $\left.{ }^{+} 01\right]$ Daum, M.; Klein, A.; Leimeister, J. M.; Krcmar, H.: Webbasierte Informationsund Interaktionsangebote für Onkologiepatienten - Ein Überblick. Arbeitspapier Nr. 109. Universität Hohenheim, Lehrstuhl für Wirtschaftsinformatik, Hohenheim 2001.

[Deut03] Deutsche Krebshilfe: Krebs: Zahlen, Daten, Fakten. http://www.krebshilfe.de/ neu/medieninfos/zahlen_daten_fakten.htm, zugegriffen am 29.10.2003

[Dier ${ }^{+}$03] Dierks, C.; Nitz, G; Grau, U.: Gesundheitstelematik und Recht. Frankfurter Schriften, MedizinRecht.de-Verlag, Frankfurt a. M. 2003.

[Döri03] Döring, N.: Sozialpsychologie des Internet : die Bedeutung des Internet für Kommunikationsprozesse, Identitäten, soziale Beziehungen und Gruppen. 2. Auflage, Internet und Psychologie, Hogrefe, Göttingen 2003.

[Ebne ${ }^{+}$03] Ebner, W.; Leimeister, J. M.; Krcmar, H.: Vertrauen in virtuellen Communities: Konzeption und Umsetzung vertrauensunterstützender Komponenten in der Domäne Healthcare. In: Proceedings of the 6. Internationale Tagung Wirtschaftsinformatik 2003: Medien - Märkte - Mobilität, S. 619-638, Dresden.

[Fogg ${ }^{+}$02] Fogg, B. J.; Soohoo, C.; Danielson, D.; Marable, L.; Stanford, J.; Tauber, E. R.: How Do People Evaluate a Web Site's Credibility? Results from a Large Study. In: http://www.consumerwebwatch.org/news/report3_credibilityresearch/stanfordPTL.pdf, zugegriffen am 10.11.2003, Stanford University, Consumer WebWatch, Sliced Bread Design, LLC. 
[Forb01] Forbiger, A.: Leben ist, wenn man trotzdem lacht. Heyne, München 2001.

[Hill01] Hiller, B.: Unkonventionelle Verfahren in der Onkologie: der Informationsbedarf der Anrufer beim Krebsinformationsdienst im Deutschen Krebsforschungszentrum zu Methoden mit bisher unbewiesener Wirksamkeit, Dissertation, Universität Heidelberg 2001.

[Hind $\left.{ }^{+} 95\right]$ Hinds, C.; Streater, A.; Mood, D.: Functions and preferred methods of receiving information related to radiotherapy: perceptions of patients with cancer. In: Cancer Nursing, (1995), Nr. 18, S. 374-384.

$\left[\mathrm{Kami}^{+}\right.$01] Kaminski, E.; Thomas, R. J.; Charnley, S.; Mackay, J.: Measuring patients response to received information. In: European Journal of Cancer, Vol. 37 (2001), Nr. Supplement 6, S. 387.

[LeSc01] Lechner, U.; Schmid, B. F.: Communities - Business Models and System Architectures: The Blueprint of MP3.com, Napster and Gnutella Revisited. In: Proceedings of the Hawaiian International Conference on System Sciences (HICSS), Hawaii.

[Leim04] Leimeister, J. M.: Pilotierung virtueller Communities im Gesundheitsbereich Bedarfsgerechte Entwicklung, Einführung und Betrieb. Dissertation, Universität Hohenheim 2004.

[LeRu92] Levenson, R. W.; Ruef, A. W.: Empathy: A psychological substrate. In: Journal of Personality and Social Psychology, Vol. 63 (1992), Nr. 2, S. 234-246.

[Lieb $\left.{ }^{+} 03\right]$ Lieberman, M.et al: Electronic support groups for breast carcinoma: a clinical trial of effectiveness. In: Cancer, Vol. 4 (2003), Nr. 97, S. 920-925.

[Mada97] Madara, E. J.: The mutual-aid self-help online revolution. In: Social Policy, Vol. 27 (1997), Nr. 3, S. 20-27.

[Mana ${ }^{+}$02] Manaszewicz, R.; Williamson, K.; McKemmish, S.: Breast Cancer Knowledge Online: Towards Meeting the Diverse Information Needs of the Breast Cancer Community. In: Proceedings of the Electronic Networking - Building Community.

[Mayh92] Mayhew, D. J.: Principles and guidelines in software user interface design. Prentice Hall, Englewood Cliffs 1992.

[McTh95] McCaughan, E. M.; Thompson, K. A.: Issues in patient care: information needs of cancer patients receiving chemotherapy at a day-case unit in Northern Ireland. In: Cancer Nursing, Vol. 18 (1995), Nr. 5, S. 374-384.

[ $\left.\mathrm{McPh}^{+} 01\right]$ McPherson, C. J.; Higginson, I. J.; Hearn, J.: Effective methods of giving information in cancer: a systematic literature review of randomized controlled trials. In: Journal of Public Health Medicine, Vol. 23 (2001), S. 227-234.

[MiSu99] Mills, M. E.; Sullivan, K.: The importance of information giving for patients newly diagnosed with cancer: a review of the literature. In: Journal of Clinical Nursing, Vol. 8 (1999), Nr. 6, S. 631-642.

[Moel96] Moeller, M. L.: Selbsthilfegruppen: Anleitungen und Hintergründe. RororoSachbuch : Psychologie aktiv, Rowohlt, Reinbek bei Hamburg 1996. 
[Niel02] Nielsen, J.: Coordinating user interfaces for consistency (Nachdruck von 1989. Auflage). San Francisco 2002.

[NoPr00] Nonnecke, B.; Preece, J.: Lurker Demographics: Counting the Silent. In: Proceedings of the ACM CHI 2000, S. 73-80, The Hague, NL.

[PeRo97] Peppers, D.; Rogers, M.: Enterprises One to One: Tools for Competing the Interactive Age. New York 1997.

[Pree99] Preece, J.: Emphatic communities: balancing emotional and factual communication. In: Interacting with Computers, Vol. 12 (1999), S. 63-77.

[Pree00] Preece, J.: Online Communities - Designing Usability, Supporting Sociability. John Wiley and Sons, Chichester, New York, 2000.

[PrMa03] Preece, J.; Maloney-Krichmar, D.: Online Communities: Focussing on Sociability and Usability. In: Handbook of Human-Computer Interaction. Hrsg.: Jacko, J.; Sears, A., Lawrence Earlbaum Associates Inc., Mahwah (NJ) 2003.

[RaLa93] Rafaeli, S.; LaRose, R. J.: Electronic Bulletin Boards and "Public Goods" - Explanations of Collaborative Mass Media. In: Communication Research, Vol. 20 (1993), Nr. 2, S. 277-290.

[Rupr98] Ruprecht, T. M.: Experten fragen - Patienten antworten : Patientenzentrierte Qualitätsbewertung von Gesundheitsdienstleistungen: Konzepte, Methoden, praktische Beispiele. Asgard-Verlag, Sankt Augustin 1998.

[Schu99] Schubert, P.: Virtuelle Transaktionsgemeinschaften im Electronic Commerce: Management, Marketing und Soziale Umwelt. Electronic Commerce, Josef Eul Verlag, Lohmar, Köln 1999.

[SEKI00] SEKIS: Nutzeranliegen an Patienteninformation - Auswertung des Expertendialogs und der Fragebogenerhebung zum Informationsbedarf aus Patientensicht. SEKIS Selbsthilfe Kontakt- und Informationsstelle, Berlin 2000.

[SeUd93] Semmer, N.; Udris, I.: Bedeutung und Wirkung von Arbeit. In: Lehrbuch Organisationspsychologie. Hrsg.: Schuler, H., Hans Huber Verlag, Bern 1993.

[Spol01] Spolsky, J.: User interface design for programmers. Apress, Berkeley 2001.

[WaKr99] Ward, S.; Kroll, P.: Building Web Solutions with the Rational Unified Process: Unifying the Creative Design Process and the Software Engineering Process. In: http://www.rational.com/media/whitepapers/76.pdf, zugegriffen am 10.11.2003

[WEBf03a] WEBforALL: Die Bedeutung des Internets für Behinderte. In: http://www.webforall.info/html/deutsch/bedeutung_des_internet.php, zugegriffen am 10.11.2003, VbI e.V. - Vereins zur beruflichen Integration und Qualifizierung e.V.

[WEBf03b] WEBforALL: Empfehlungen für ein barrierefreies Webdesign. http://webforall.info/html/deutsch/empfehlungen.php, zugegriffen am 10.11.2003, VbI e.V. - Vereins zur beruflichen Integration und Qualifizierung e.V.

[WiLi01] Wirtz, B. W.; Lihotzky, N.: Internentökonomie, Kundenbindung und Protalstrategien. In: Die Betriebswirtschaft, Vol. 61 (2001), Nr. 3, S. 285-305. 